\title{
DARK STARS: IMPROVED MODELS AND FIRST PULSATION RESULTS
}

\author{
T. Rindler-Daller ${ }^{1}$, M. H. Montgomeri ${ }^{2}$, K. Freese $^{1}$, D. E. Winget ${ }^{2}$, and B. Paxton ${ }^{3}$ \\ ${ }^{1}$ Department of Physics and Michigan Center for Theoretical Physics, University of Michigan, Ann Arbor, MI 48109, USA \\ ${ }^{2}$ Department of Astronomy, McDonald Observatory and Texas Cosmology Center, University of Texas, Austin, TX 78712, USA \\ ${ }^{3}$ Kavli Insitute for Theoretical Physics, University of California, Santa Barbara, CA 93106, USA \\ Received 2014 August 9; accepted 2014 November 25; published 2015 January 30
}

\begin{abstract}
We use the stellar evolution code MESA to study dark stars (DSs). DSs, which are powered by dark matter (DM) selfannihilation rather than by nuclear fusion, may be the first stars to form in the universe. We compute stellar models for accreting DSs with masses up to $10^{6} M_{\odot}$. The heating due to DM annihilation is self-consistently included, assuming extended adiabatic contraction of DM within the minihalos in which DSs form. We find remarkably good overall agreement with previous models, which assumed polytropic interiors. There are some differences in the details, with positive implications for observability. We found that, in the mass range of $10^{4}-10^{5} M_{\odot}$, our DSs are hotter by a factor of 1.5 than those in Freese et al., are smaller in radius by a factor of 0.6 , denser by a factor of three to four, and more luminous by a factor of two. Our models also confirm previous results, according to which supermassive DSs are very well approximated by $(n=3)$-polytropes. We also perform a first study of DS pulsations. Our DS models have pulsation modes with timescales ranging from less than a day to more than two years in their rest frames, at $z \sim 15$, depending on DM particle mass and overtone number. Such pulsations may someday be used to identify bright, cool objects uniquely as DSs; if properly calibrated, they might, in principle, also supply novel standard candles for cosmological studies.
\end{abstract}

Key words: astroparticle physics - dark ages, reionization, first stars - dark matter - stars: evolution stars: oscillations (including pulsations)

\section{INTRODUCTION}

The first stars are thought to form at redshifts of $z \sim 15-30$ inside dark matter (DM) minihalos of mass $\sim 10^{6}-10^{8} M_{\odot}$, which consist of $85 \%$ DM and $15 \%$ baryons, mostly in the form of hydrogen and helium from big bang nucleosynthesis. The formation of the first stars is currently a hot topic in astrophysical cosmology (for more on the formation of the first stars, see, e.g., Barkana \& Loeb 2001; Abel et al. 2002; Yoshida et al. 2003; Bromm \& Larson 2004; Alvarez et al. 2006; Ahn \& Shapiro 2007; Turk et al. 2009; Hosokawa et al. 2011; Greif et al. 2012; Smith et al. 2012a). Up-to-date reviews can be found, e.g., in Bromm (2013) and Glover (2013).

Early and most subsequent investigations have largely neglected the impact of self-annihilating DM on the chemistry and physics of primordial protostellar clouds, and hence on first star formation. The canonical DM candidates, weakly interacting massive particles (WIMPs), in many theories, are their own antiparticles and able to annihilate with one another. This annihilation process in the early universe can leave the correct relic abundance today. In addition, annihilation can be important wherever the DM density $\rho_{\chi}$ is high, since the annihilation rate scales as $\rho_{\chi}^{2}$.

The formation of the first stars is expected to be particularly affected by this process, since they form at high redshifts $\left(\rho_{\chi} \sim(1+z)^{3}\right)$ and in the high-density centers of DM minihalos. Spolyar et al. (2008) were the first to consider the effect of DM particles on the first stars during their formation. They found that, above a certain baryonic density threshold (the value of which depends on the DM particle mass) heating by DM annihilation will come to dominate over all cooling mechanisms. Subsequently, the protostellar cloud will continue to contract, albeit at a slower rate, and eventually, above a certain baryonic density threshold (again depending on the DM particle mass), DM annihilation products remain trapped in the star, thermalize and provide a heat source for hydrostatic equilibrium: a dark star (DS) is born. These first DSs are made primarily of hydrogen and helium, with less than $0.1 \%$ of the mass in form of DM. Nevertheless, they shine due to DM heating, not fusion, and so the term "dark" refers to the power source, and not the appearance or the primary matter constituent. Subsequently, Freese et al. (2008a) and Spolyar et al. (2009) studied the evolution of DSs from their birth at $\sim 1 M_{\odot}$, as they accreted material from the surrounding halo, up to $\sim 1000 M_{\odot}$, which is approximately the Jeans mass of the collapsing molecular cloud. They showed that DSs are giant, puffy ( $\sim 10 \mathrm{AU})$, cool $\left(T_{\text {eff }}<10,000 \mathrm{~K}\right)$, and bright $\left(>10^{6} L_{\odot}\right)$ objects. Since their surface temperatures never exceed values that are high enough to trigger feedback mechanisms that would shut off further accretion (see Tan \& McKee 2004), DSs can in principle grow as long as there is a supply of DM fuel. Indeed, Freese et al. (2010) followed the growth of DSs to become supermassive with masses in excess of $10^{5} M_{\odot}$. These supermassive DSs are extraordinarily luminous, $L \sim 10^{9}-10^{11} L_{\odot}$, and may be observable with upcoming facilities (see Freese et al. 2010; Ilie et al. 2012).

The WIMP annihilation rate is $n_{\chi}^{2}\langle\sigma v\rangle$, where $n_{\chi}$ is the WIMP number density and we take the standard annihilation cross section

$$
\langle\sigma v\rangle=3 \times 10^{-26} \mathrm{~cm}^{3} \mathrm{~s}^{-1},
$$

and WIMP masses in the range $m_{\chi}=10 \mathrm{GeV}-1 \mathrm{TeV}$. WIMP annihilation produces energy at a rate per unit volume

$$
\hat{Q}_{\mathrm{DM}}=n_{\chi}^{2}\langle\sigma v\rangle m_{\chi}=\langle\sigma v\rangle \rho_{\chi}^{2} / m_{\chi},
$$

where $\rho_{\chi}$ is the WIMP mass density. We note the dependence of the DM heating $\hat{Q}_{\mathrm{DM}} \propto\langle\sigma v\rangle / m_{\chi}$, so that by studying a wide range of WIMP masses we are effectively studying a comparable range of annihilation cross sections. 
The annihilation products typically are electrons, photons, and neutrinos. The neutrinos escape the star, while the other annihilation products are trapped in the dark star, thermalize with the star, and heat it up. The luminosity from the DM heating is

$$
L \sim f_{Q} \int \hat{Q}_{\mathrm{DM}} d V,
$$

where $f_{Q}$ is the fraction of the annihilation energy deposited in the star (not lost to neutrinos) and $d V$ is the volume element. We take $f_{Q}=2 / 3$ as is typical for WIMPs, see Spolyar et al. (2008).

Spolyar et al. (2009) and Freese et al. (2010) consider two mechanisms for supplying DM "fuel." One is gravitational contraction in which DM is supplied by the gravitational attraction of baryons in the star. We label this mechanism AC for adiabatic contraction (see Blumenthal et al. 1986), the technique that allows us to calculate the resultant DM density inside the star. This is a generic process and is expected to occur in the halos in which DSs form. It has been shown in Freese et al. (2009) that the choice of initial DM profile, as well as details in the assumption of the DM orbits involved, is not crucial, and the effect prevails. Spolyar et al. (2009) found the following approximation on how the DM density follows the (baryonic) gas density $n_{h}$, namely,

$$
\rho_{\chi} \sim 5\left(\mathrm{GeV} / \mathrm{cm}^{-3}\right)\left(n_{h} / \mathrm{cm}^{3}\right)^{0.81} .
$$

In this paper, we implement AC, following Spolyar et al. (2009) and Freese et al. (2010), using the Blumenthal method. ${ }^{4}$

The second mechanism refers to replenishing of DM inside the star by capture of WIMP DM from the surroundings as it scatters elastically off of nuclei in the star (see Freese et al. 2008b; Iocco 2008). This elastic scattering is the same mechanism that is searched for in direct WIMP detection experiments. In both fueling mechanisms, the final stellar mass is driven to be very high (with and without capture), and while DM reigns, the star remains bright but cool. The DSs growing via WIMPs captured via elastic scattering are hotter and denser than the ones formed via $\mathrm{AC}$ alone. In this paper, we restrict our studies to WIMPs gravitationally brought in via $\mathrm{AC}$ alone and do not consider captured DM.

The main focus of this paper is to improve upon the modeling of the stellar evolution of DSs. The calculations of Freese et al. (2008a); Spolyar et al. (2009); Freese et al. (2010) were based on the assumption of polytropic stellar interiors. More precisely, as soon as the conditions were ripe for DS formation $\left(M \sim 1-10 M_{\odot}\right)$, DSs were built up by accretion of their surrounding material, while an iterative procedure ensured that polytropic equilibrium configurations were found along the evolutionary sequence.

Instead of polytropic models, our current work employs the fully fledged 1D stellar evolution code MESA, ${ }^{5}$ which allows us to solve the stellar structure equations self-consistently, without restrictive assumptions on the equation of state or overall structure of the stellar models. This is accomplished through an additional module in MESA that locally adds the energy due to DM heating.

Using these models, we are also able to study deviations from equilibrium, which allows us to explore the question of DS pulsations. We accomplish this at the expense of neglecting

\footnotetext{
4 We do not use the simple formula given in Equation (4).

5 http://mesa.sourceforge.net/
}

some physical effects in this work. While we do implement extended AC and DM heating self-consistently, we do not include DM capture or nuclear burning, in contrast to Spolyar et al. (2009) and Freese et al. (2010). However, now that we are comfortable that our newly implemented module in MESA is working successfully, i.e., giving robust results, regardless of initial stellar mass, WIMP mass, or halo environment, work is in progress to include the above effects in a future publication.

There have been some recent critiques of the idea of DSs. While a full response is not appropriate here, we will mention a few points; a more detailed account can be found in a response published on the arXiv (see Gondolo et al. 2013).

First, previous simulations such as those in Ripamonti et al. (2010) and Smith et al. (2012b) have studied collapsing protostellar clouds and noted that the collapse continues past a hydrogen density that is higher than the one quoted in Spolyar et al. (2008) (the exact value of this threshold depends on the adopted WIMP mass). This fact led to the incorrect conclusion that DM heating is not potent enough for the establishment of a hydrostatic equilibrium of a DM heating-powered DS. However, subsequent work by Freese et al. (2008a) and Spolyar et al. (2009) has shown that the expected DM densities in models of fully formed DSs, which are supported by DM annihilation, are indeed much higher. Due to resolution limits, the aforementioned simulations are unable to reach densities this high and are therefore unable to directly address the DS regime.

Second, a growing body of literature finds that the central accretion disk around the protostar can fragment (see Stacy et al. 2010; Clark et al. 2011; Greif et al. 2012; Stacy et al. 2014). This could lead to the formation of multiple protostars, and the removal of a central object from the DM cusp. Furthermore, these protostars (see Stacy et al. 2012) could gravitationally scatter the DM and thereby remove it from the central DM cusp. Of course, the latter study presupposes the presence of multiple protostars, and therefore assumes that DM heating does not prevent fragmentation of the disk. However, Smith et al. (2012b) have found that the inclusion of DM heating can actually help stabilize the protostellar disk, preventing fragmentation around the central protostar within a radius of about $1000 \mathrm{AU} \sim 2 \times 10^{5} R_{\odot}$ within $\sim 6100 \mathrm{yr}$ after formation of the primary protostar in the center. This in turn could prevent the formation of multiple protostars and their subsequent gravitational scattering of DM from the center.

We note that the simulations can only follow the evolution for so long by adopting sink particles to those regions that approach stellar densities. Hence, it is plausible that a DS could form in the central sink particle of the Smith et al. (2012b) simulations, at densities that are much higher and at radii that are much smaller than can be resolved, as noted by Smith et al. (2012b). However, the DM is "locked in" to the central protostar in Smith et al. (2012b) and does not dynamically follow the movement of the baryons. Future studies with high enough resolution to capture the mutual dynamical effects of baryons and DM in those very central regions will be necessary in order to assess the feasibility of the formation of DSs. In the meantime, we have good reasons for assuming that DSs can form, and the final arbiter for their existence will be any distinct observational signatures that they possess. To this end, in this paper we present improved, state-of-the-art calculations of the observable parameters of DSs. As with nearby stars, studying their evolution is a much simpler problem than describing the formation process itself. 


\section{TREATMENT OF STELLAR EVOLUTION}

The stellar evolution calculations in this paper were performed using the open source software package MESA ("Modules for Experiments in Stellar Astrophysics"; Paxton et al. 2011). This flexible and state-of-the-art one-dimensional evolution code can be used to treat a wide variety of problems, from main sequence evolution, to the red giant and asymptotic giant phases, and finally to the white dwarf phase. Additionally, it can be applied to models that are accreting or losing mass. Lately, MESA has been significantly updated and advanced in its capabilities to model the evolution of giant planets, low-mass stars, massive stars, along with additional stellar features such as rotation and asteroseismology (Paxton et al. 2013). MESA is regularly upgraded, as a result of continuous feedback by the community of MESA users and their differing scientific needs. In fact, the distinctive physics of rapidly growing DSs in a primordial environment is one example of a problem that can easily challenge a stellar evolution code, which may otherwise be optimized to the more standard evolution of stars in our local universe.

In MESA, material that is accreted is set to have the same entropy as the surface layers of the model. Thus, accretion does not directly heat the surface. This is consistent with a physical picture in which material passes through an accretion disk, gradually radiating away the gravitational energy of its infall. By contrast, spherical accretion involves the formation of a shock front at the stellar surface, which increases the entropy of the accreted material and heats the surface layers. By examining both mechanisms for the growth of massive protostars, Hosokawa et al. (2010) find that their models converge to the same radii and temperatures as a function of mass for masses $\gtrsim 40 M_{\odot}$. In addition, given the larger radii of our models, the gravitational energy release of any infalling matter should be even less important. For these reasons, we use the default prescription in MESA for accretion.

In order to study the effect of DM self-annihilation heating in stars, we have used MESA's ' 'other_energy_implicit", interface to add a DM heating module to MESA (see Appendix B for details). DM annihilation provides a powerful heat source in the first stars. As long as the central temperature of a star in equilibrium is below the onset of nuclear fusion, DM heating will be the only heat source in that star, solely responsible for its luminosity. We shall clarify here some terms: in the initial contracting phase of a protostellar cloud, heat is released by means of gravitational contraction, which is given by the Kelvin-Helmholtz timescale of the protostar. Usually, this whole process by which the newborn star settles into a quasi-equilibrium up to the final onset of nuclear burning in the star's center is called the pre-main-sequence phase. In the absence of DM heating, this phase usually lasts much shorter than the main-sequence phase of nuclear burning.

In the presence of DM heating, however, DSs can accrete substantial amounts of mass and yet stay cool enough for nuclear fusion to be delayed; we are confirming this scenario in this paper. In fact, in accordance with Freese et al. (2010), we find that DSs residing in the centers of their host halos can grow to supermassive size of $10^{4-6} M_{\odot}$, and this process can take $10^{5-9} \mathrm{yr}$, depending on the accretion rate, and assuming a continuous fuel of DM is provided. Once the DM fuel runs out, the supermassive DS may run through a rapid sequence of changes, whereby it shrinks, seeking for a new equilibrium, until the central temperature is high enough for fusion to start.
A fusion-powered star that massive, however, cannot survive for long, and the DS may collapse to form a massive black hole soon after. Indeed, this way, DSs could provide a compelling cause for the early formation of supermassive black holes, which have been observed in the centers of galaxies in the local, as well as in the high-redshift universe. Thus, massive DSs spend most of their lives in the pre-fusion phase, and hence the term "pre-mainsequence" phase is a misnomer in the case of DSs. Therefore, we will try to avoid this term, noting, though, that the preparation of the initial conditions in MESA are accomplished with a module of that name (see also the next subsection).

\subsection{Initial Conditions}

In MESA a new evolution can be started by creating a "premain-sequence model" upon specifying the mass, a uniform composition, a luminosity, and a central temperature $T_{c}$ low enough to prevent nuclear burning. For a fixed $T_{c}$ and composition, the total mass depends only on the central density, $\rho_{c}$. An initial guess for $\rho_{c}$ is made by using an $n=3 / 2$ polytrope, appropriate for a fully convective star, although MESA does not assume that the star is fully convective during the subsequent search for a converged pre-main-sequence model. Instead, MESA uses its routines for solving the equations of stellar structure, equation of state, and "mixing-length theory" (MLT) for the treatment of convection in order to search for a $\rho_{c}$ that gives the model of the desired mass. That initial guess may not be optimum for DSs, but our MESA models tend to converge quickly toward equilibrium sequences.

In light of the comparison of our results with previous polytropic models of supermassive DSs, we use the same parameters for the halo environment as in Freese et al. (2010). According to their choice, we consider models of DSs which are accreting matter at a (constant) rate of $\dot{M}=10^{-3} M_{\odot} \mathrm{yr}^{-1}$ in a host minihalo of $10^{6} M_{\odot}$, forming at a redshift of $z=20$, as well as at a higher rate of $M=10^{-1} M_{\odot} \mathrm{yr}^{-1}$ in a larger host halo of $10^{8} M_{\odot}$ with a formation redshift of $z=15$, respectively. As our terminology throughout the paper, we use

$$
\begin{aligned}
& \text { SMH }: \dot{\mathrm{M}}=10^{-3} M_{\odot} \mathrm{yr}^{-1},\left(\text { host halo of } 10^{6} M_{\odot}\right) \\
& \mathrm{LMH}: \dot{\mathrm{M}}=10^{-1} M_{\odot} \mathrm{yr}^{-1},\left(\text { host halo of } 10^{8} M_{\odot}\right) .
\end{aligned}
$$

For both halo masses, we choose a fraction of $15 \%$ baryons and $85 \% \mathrm{DM}$, a primordial metallicity of $Z=0$, and a hydrogen-tohelium fraction of 0.76 . For each halo, we assume that initially both the baryons and the DM can be described with the same NFW density profile (see Navarro et al. 1996),

$$
\rho(r)=\frac{\rho_{0}}{r / r_{s}\left(1+r / r_{s}\right)^{2}},
$$

where $\rho_{0}$ is the central density and $r_{s}$ is the scale radius. At any point of the profile, baryons will only make up $15 \%$ of the mass. The density scale, $\rho_{0}$ can be reexpressed in terms of the critical density of the universe at a given redshift, $\rho_{c}(z)$ via

$$
\rho_{0}=\rho_{c}(z) \frac{178}{3} \frac{c^{3}}{\ln (1+\mathrm{c})-\mathrm{c} /(\mathrm{c}+1)},
$$

where $c \equiv r_{\mathrm{vir}} / r_{s}$ is the concentration parameter and $r_{\mathrm{vir}}$ is the virial radius of the halo. We choose a fiducial value of $c=3.5$. In fact, the properties of DSs stay roughly the same for concentration parameters $c=2-5$, as has been shown in Ilie 

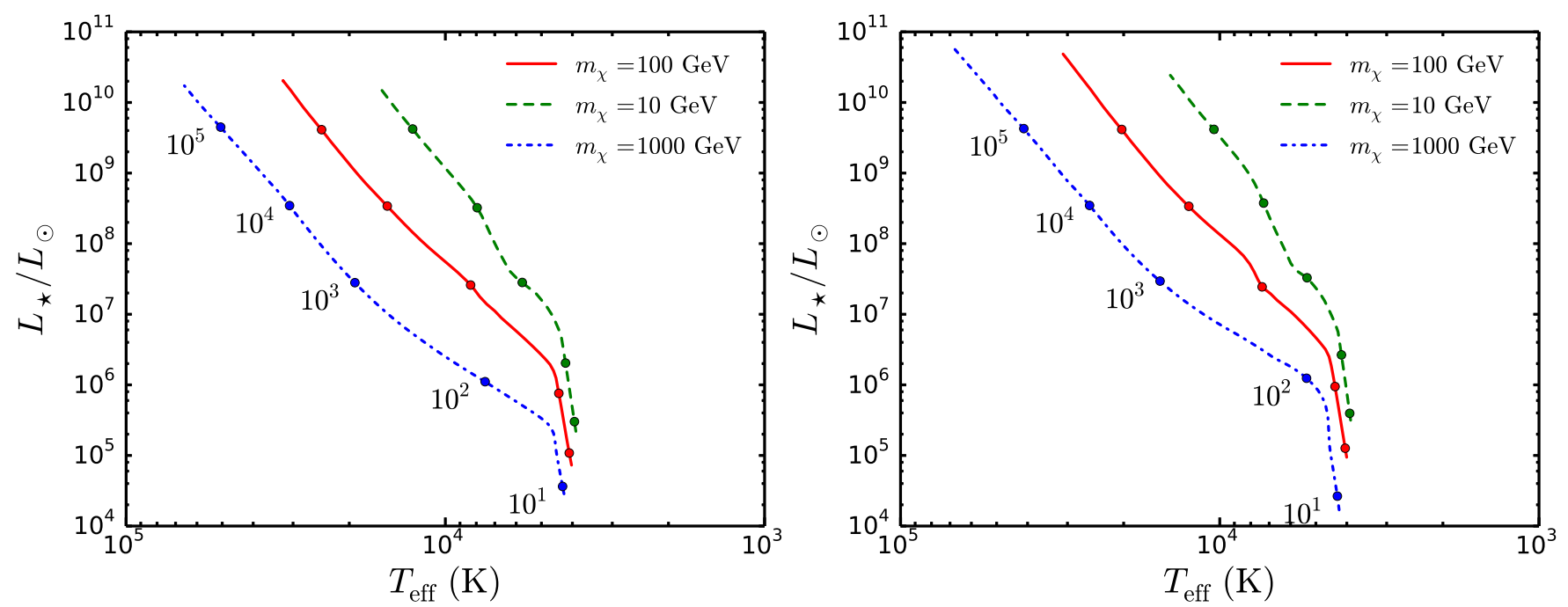

Figure 1. Evolution of dark stars in a host minihalo of mass $10^{6} M_{\odot}$ ("small minihalo"-SMH) with an accretion rate of $\dot{M}=10^{-3} M_{\odot} \mathrm{yr}^{-1}$ (left-hand plot), and evolution of dark stars in a host halo of mass $10^{8} M_{\odot}$ ("large minihalo"-LMH) with an accretion rate of $\dot{M}=10^{-1} M_{\odot} \mathrm{yr}^{-1}$ (right-hand plot), respectively, for WIMP masses of 10,100, and $1000 \mathrm{GeV}$. The highlighted dots on the tracks correspond to the benchmark values of the dark star mass, according to Tables 1 and 2, respectively. The calculations assume extended adiabatic contraction and no significant depletion of dark matter due to annihilation. Dark matter capture is not considered.

et al. (2011). While cosmological $N$-body simulations suggest that the profile in Equation (7) is a good fit to most DM halos, there is still uncertainty about the exact inner density slope of DM halos. It has been shown in Freese et al. (2009), however, that DSs result, regardless of the inner density profile; that paper considered even the extreme case of a cored Burkert profile and found the resulting DSs. We assume a flat $\Lambda$ CDM universe, using the cosmological parameters from the three-year data of the WMAP satellite (Spergel et al. 2007), i.e., a present matter density $\Omega_{m}=0.24$, Hubble parameter $h=0.732$, and hence a dark energy density of $\Omega_{\Lambda}=0.76$. These are the parameters used in previous works on DS evolution, and for the sake of comparison, we will use these parameters for our MESA calculations, as well. ${ }^{6}$

For models with WIMP masses higher than $m_{\chi}=10 \mathrm{GeV}$, we choose an initial stellar mass of $2 M_{\odot}$. For models with $m_{\chi}=10 \mathrm{GeV}$, we need higher initial masses for the initial models to converge. In those cases, we chose an initial mass of $5 M_{\odot}$.

\section{EVOLUTION OF SUPERMASSIVE DARK STARS}

We explore three different values of the mass of the DM particles, 10, 100, and $1000 \mathrm{GeV}$, and for each we compute a sequence of DS models starting at $2 M_{\odot}$ (or $5 M_{\odot}$ ) and ending at over $10^{5} M_{\odot}$ for the minihalo ("SMH"), and $10^{6} M_{\odot}$ for the larger halo ("LMH"), respectively. ${ }^{7}$ Some comments are in order here before we proceed to show the results. The protostellar

\footnotetext{
6 We performed test runs to see how DS properties are affected by using more recent WMAP or Planck satellite data (Ade et al. 2014), respectively. The changes are too small to be noticable on the plots presented in this paper. A change of the values for the cosmological parameters of the order of $\sim 10 \%$ will thus not affect our basic results.

7 For notational convenience, we may call the $10^{8} M_{\odot}$ halo (LMH) sometimes a "minihalo" as well, even though this runs counter to the definition of minihalos as objects with virial temperatures lower than about $10,000 \mathrm{~K}$, which sets the boundary above which atomic cooling prevails over molecular cooling. The mass range of minihalos is redshift dependent: for our chosen redshifts, the maximum mass of minihalos amounts to about $1.3 \times 10^{7} M_{\odot}$ at $z=20$ and $2 \times 10^{7} M_{\odot}$ at $z=15$, respectively, according to Equation (2) in Shapiro et al. (2004).
}

accretion rate can be estimated from the free-fall timescale of the Jeans mass of the gas, as

$$
\dot{M} \simeq \frac{M_{J}}{t_{\mathrm{ff}}} \simeq \frac{c_{s}^{3}}{G} \propto T^{3 / 2}
$$

i.e., accretion rates are substantially higher in the hotter primordial star formation clouds, compared to those in the present universe, which have more efficient ways of cooling. The above choice of values for $\dot{M}$, Equations (5) and (6), is related to the adopted size of minihalo, since the ambient temperature is expected to be higher in larger minihalos, leading to higher accretion rates. It shall be emphasized that values of $\dot{M}$ in excess of $\gtrsim 10^{-3} M_{\odot} \mathrm{yr}^{-1}$ are actually very high, and present a challenge to the numerical capabilities of stellar evolution codes. In fact, after initial difficulties, we were able to calculate models with accretion rates up to $10^{-1} M_{\odot} \mathrm{yr}^{-1}$, using an upgraded version of MESA. All calculations in this paper have been performed using release 5596 of MESA.

In Figure 1, we show the location of our model sequences in the Hertzsprung-Russell diagram for each halo environment. The tracks are monotonic, once the star settles into a quasi-static equilibrium. Since the DM heating is proportional to $1 / m_{\chi}$ (see Equation (2)), we obtain different tracks for different particle masses. The heating in the $m_{\chi}=10 \mathrm{GeV}$ models is the largest, so these models have more pressure support and are therefore larger: at constant luminosity these models have cooler surface temperatures. In contrast, the $m_{\chi}=1000 \mathrm{GeV}$ models have less heating and are therefore smaller: these models are hotter at fixed luminosity. We summarize some key stellar properties for every decade of mass growth in Tables 1 and 2, respectively.

Figures 2, 8, and 9 show a comparison of the evolution of different stellar characteristics, depending on the accretion rate (Figures 8 and 9 are collected in Appendix A). By assumption of a constant accretion rate, the $M_{\star}$-age relationship is simply linear and a given age DS is more massive at higher accretion rates. For a given DS mass, the low-accretion environment (SMH) will produce stars of smaller radius, higher density and higher surface temperatures. This is true, regardless of the value for the WIMP mass. 

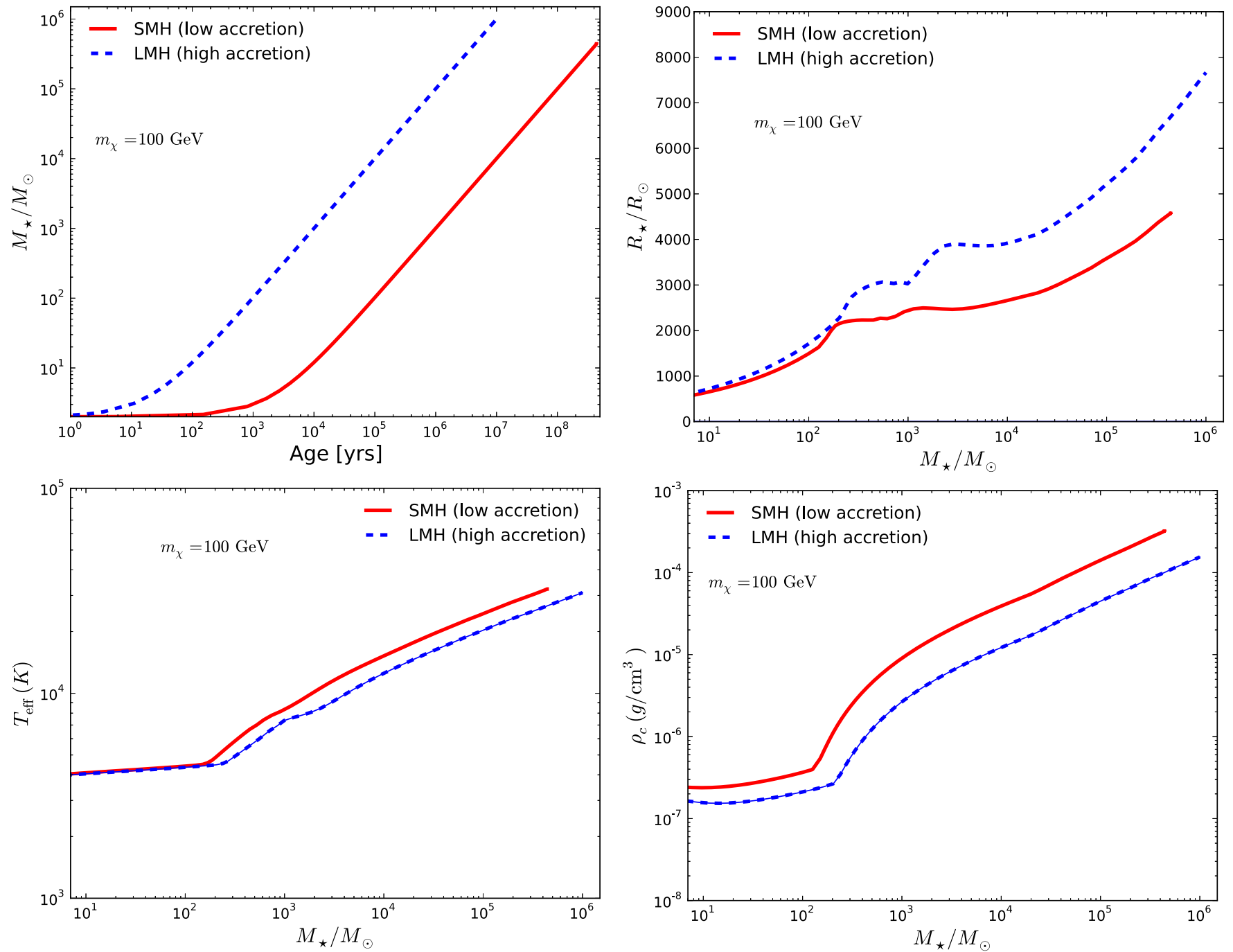

Figure 2. Evolution of dark stars, illustrating the dependence on accretion rate (SMH: $\dot{M}=10^{-3} M_{\odot} ; \mathrm{LMH}: \dot{M}=10^{-1} M_{\odot}$ ). The quantities plotted are stellar mass (upper left), stellar radius (upper right), surface temperature (bottom left), and central density (bottom right). In each case, the DM particle mass is $m_{\chi}=100 \mathrm{GeV}$. The same plots for different WIMP masses can be found in Appendix A, Figures 8 and 9.

Table 1

Properties of Dark Stars along the Evolutionary Sequence for $m_{\chi}=100 \mathrm{GeV}$ and $\dot{M}=10^{-3} M_{\odot} \mathrm{yr}^{-1}(\mathrm{SMH}):$ Mass $M_{\star}$,

Luminosity $L_{\star}$, Radius $R_{\star}$, Effective Temperature $T_{\text {eff }}$,

Central Temperature $T_{c}$, and Total Central Density $\rho_{c}$

\begin{tabular}{lrcccc}
\hline \hline $\begin{array}{c}M_{\star} \\
\left(M_{\odot}\right)\end{array}$ & $\begin{array}{c}L_{\star} \\
\left(10^{6} L_{\odot}\right)\end{array}$ & $\begin{array}{c}R_{\star} \\
\left(R_{\odot}\right)\end{array}$ & $\begin{array}{c}T_{\text {eff }} \\
\left(10^{3} \mathrm{~K}\right)\end{array}$ & $\begin{array}{c}T_{c} \\
\left(10^{5} \mathrm{~K}\right)\end{array}$ & $\begin{array}{c}\rho_{c} \\
\left(\mathrm{~g} \mathrm{~cm}^{-3}\right)\end{array}$ \\
\hline 10 & 0.11 & 655.7 & 4.1 & 1.0 & $2.4 \times 10^{-7}$ \\
100 & 0.76 & 1493.3 & 4.4 & 2.6 & $3.7 \times 10^{-7}$ \\
500 & 10.41 & 2257.2 & 6.9 & 8.6 & $4.6 \times 10^{-6}$ \\
$10^{3}$ & 25.94 & 2437.0 & 8.3 & 12.4 & $9.0 \times 10^{-6}$ \\
$10^{4}$ & 341.21 & 2659.2 & 15.2 & 30.6 & $3.9 \times 10^{-5}$ \\
$10^{5}$ & 4121.02 & 3578.6 & 24.4 & 69.5 & $1.4 \times 10^{-4}$ \\
\hline
\end{tabular}

As our DSs grow, they acquire radiation-dominated, weakly convective envelopes. In general, such regions often have large superadiabatic gradients, which can lead to slow convergence. These issues occur even for "normal" massive stars that are crossing the asymptotic giant branch. In this radiationdominated regime, stellar evolution codes experience severe numerical difficulties due to the extremely small timescales required. It is an open question whether additional physical insta-
Table 2

Properties of Dark Stars along the Evolutionary Sequence for $m_{\chi}=100 \mathrm{GeV}$ and $\dot{M}=10^{-1} M_{\odot} \mathrm{yr}^{-1}(\mathrm{LMH}):$ Stellar Quantities as in Table 1

\begin{tabular}{lrcccc}
\hline \hline $\begin{array}{c}M_{\star} \\
\left(M_{\odot}\right)\end{array}$ & $\begin{array}{c}L_{\star} \\
\left(10^{6} L_{\odot}\right)\end{array}$ & $\begin{array}{c}R_{\star} \\
\left(R_{\odot}\right)\end{array}$ & $\begin{array}{c}T_{\mathrm{eff}} \\
\left(10^{3} \mathrm{~K}\right)\end{array}$ & $\begin{array}{c}T_{c} \\
\left(10^{5} \mathrm{~K}\right)\end{array}$ & $\begin{array}{c}\rho_{c} \\
\left(\mathrm{~g} \mathrm{~cm}^{-3}\right)\end{array}$ \\
\hline 10 & 0.13 & 724.1 & 4.0 & 0.8 & $1.6 \times 10^{-7}$ \\
100 & 0.95 & 1711.0 & 4.4 & 2.2 & $2.1 \times 10^{-7}$ \\
500 & 9.28 & 3044.2 & 5.8 & 5.5 & $1.2 \times 10^{-6}$ \\
$10^{3}$ & 24.45 & 3036.4 & 7.4 & 8.2 & $2.7 \times 10^{-6}$ \\
$10^{4}$ & 338.41 & 3916.5 & 12.5 & 20.7 & $1.2 \times 10^{-5}$ \\
$10^{5}$ & 4149.34 & 5205.5 & 20.3 & 47.3 & $4.5 \times 10^{-5}$ \\
$10^{6}$ & 48203.79 & 7797.4 & 31.4 & 106.6 & $1.6 \times 10^{-4}$ \\
\hline
\end{tabular}

bilities occur in radiation-dominated stars that act to limit this superadiabaticity.

In our calculations, we encounter these difficulties as the total mass of our models approaches $10^{4} M_{\odot}$. Our approach is to use MESA's MLT++ routines to partially suppress this superadiabaticity (see Paxton et al. 2013, for more details). We found a reasonable range of parameters that not only allowed our models to grow beyond $10^{4} M_{\odot}$ but also reduced the impact caused by superadiabaticity (see also Section 4). While using MLT++ 

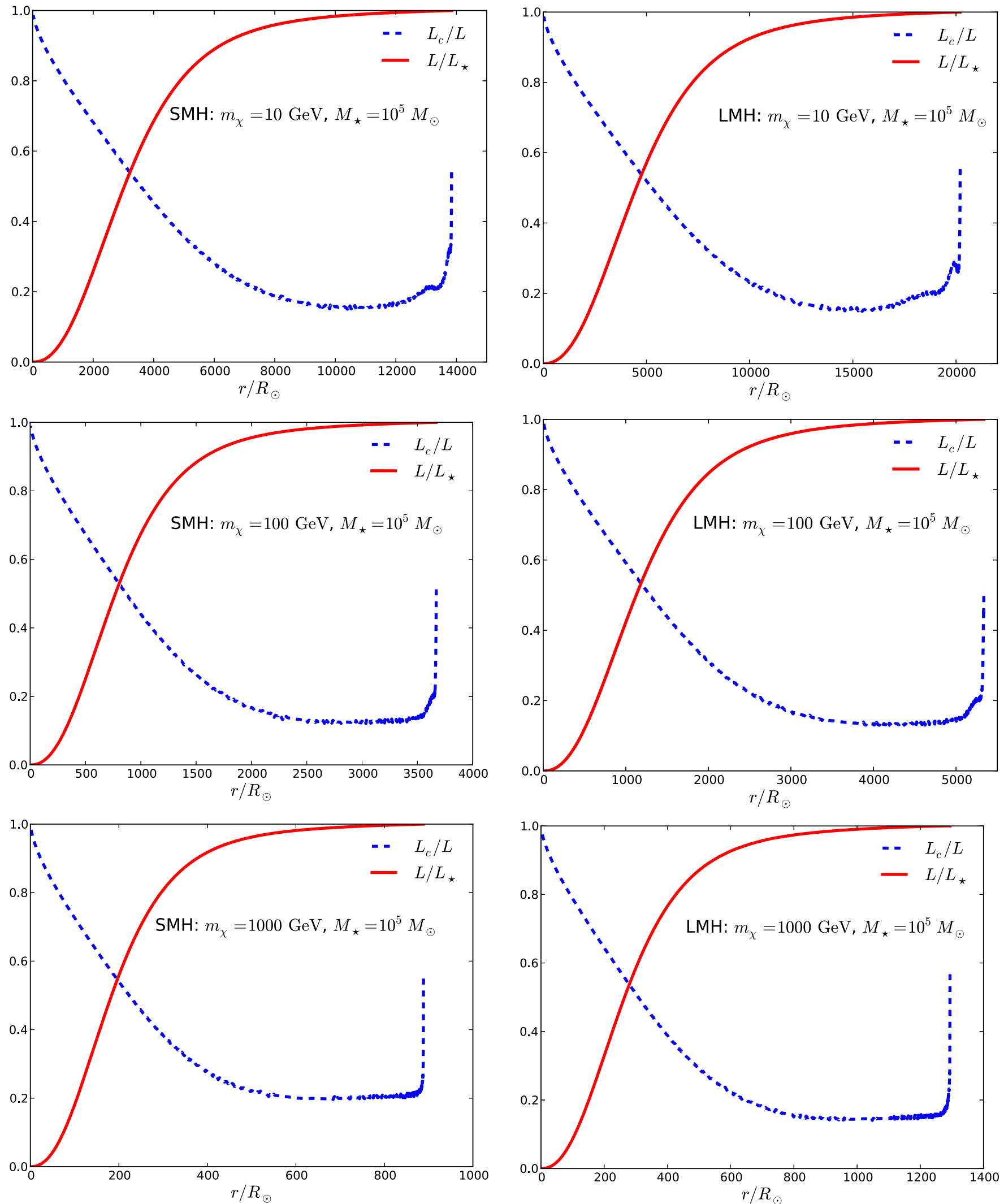

Figure 3. Luminosity at a given radius $L(r)$, as well as fraction of convective luminosity $L_{c}(r)$, as a function of radius. $L(r)$ is normalized over its value at the photosphere, $L_{\star}, L_{c}(r)$ is normalized over $L(r)$. Left column: dark star with $M_{\star}=10^{5} M_{\odot}$, forming in SMH with accretion rate $\dot{M}=10^{-3} M_{\odot} \mathrm{yr}^{-1}: \operatorname{Right}$ column: dark star with mass $M_{\star}=10^{5} M_{\odot}$ but forming in LMH with $\dot{M}=10^{-1} M_{\odot} \mathrm{yr}^{-1}$. Upper row: DM particle mass $m_{\chi}=10 \mathrm{GeV}$; Middle row: $m_{\chi}=100 \mathrm{GeV}$; Bottom row: $m_{\chi}=1000 \mathrm{GeV}$. In all cases, radiation dominates as the energy transport mechanism throughout most of the interiors of the models. 

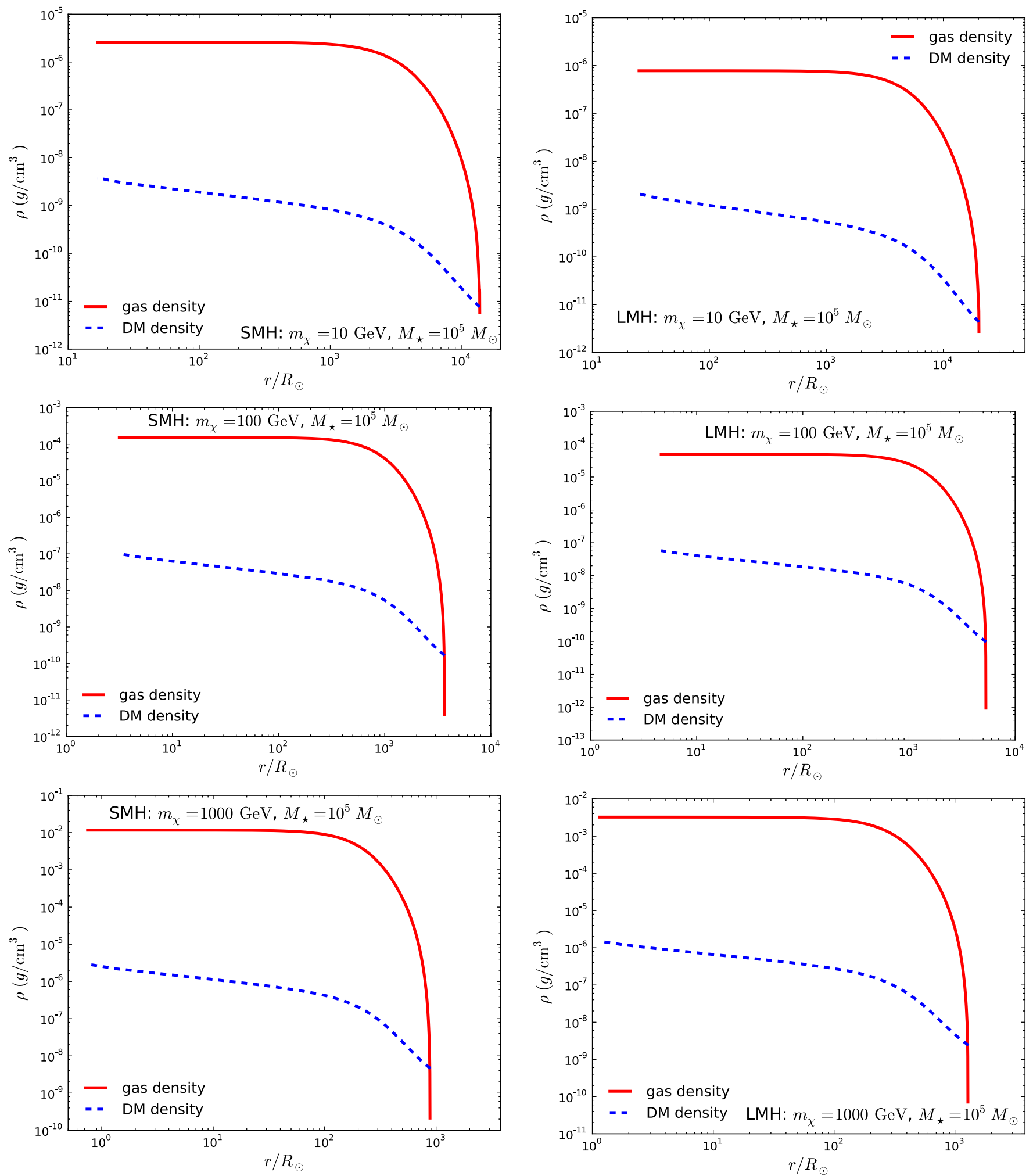

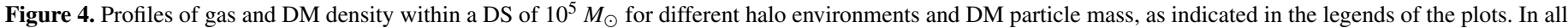
cases, the DM density is roughly three orders of magnitude below the gas density.

can change the details of the mass-luminosity relationship, it does not fundamentally or qualitatively change the behavior of the models. For instance, the "bump" in the radius at $\sim 100 M_{\odot}$ for the $1000 \mathrm{GeV}$ WIMP case in Figure 9 is caused by the sharp transition of the envelope from subadiabatic to superadiabatic. The MLT++ prescription limits this superadiabaticity, and this reduction in the temperature gradient leads to a decrease in ra- dius. Once the model settles into a new equilibrium, the radius continues its growth. Without MLT++, this feature would have been more pronounced. Similar behavior is found for all models in the mass range of $100-1000 M_{\odot}$. This transition, however, becomes less dramatic for smaller DM mass, as can be seen in Figures 2 and 8. In general, the rough features in these figures are similar signs of the onset of superadiabaticity. 

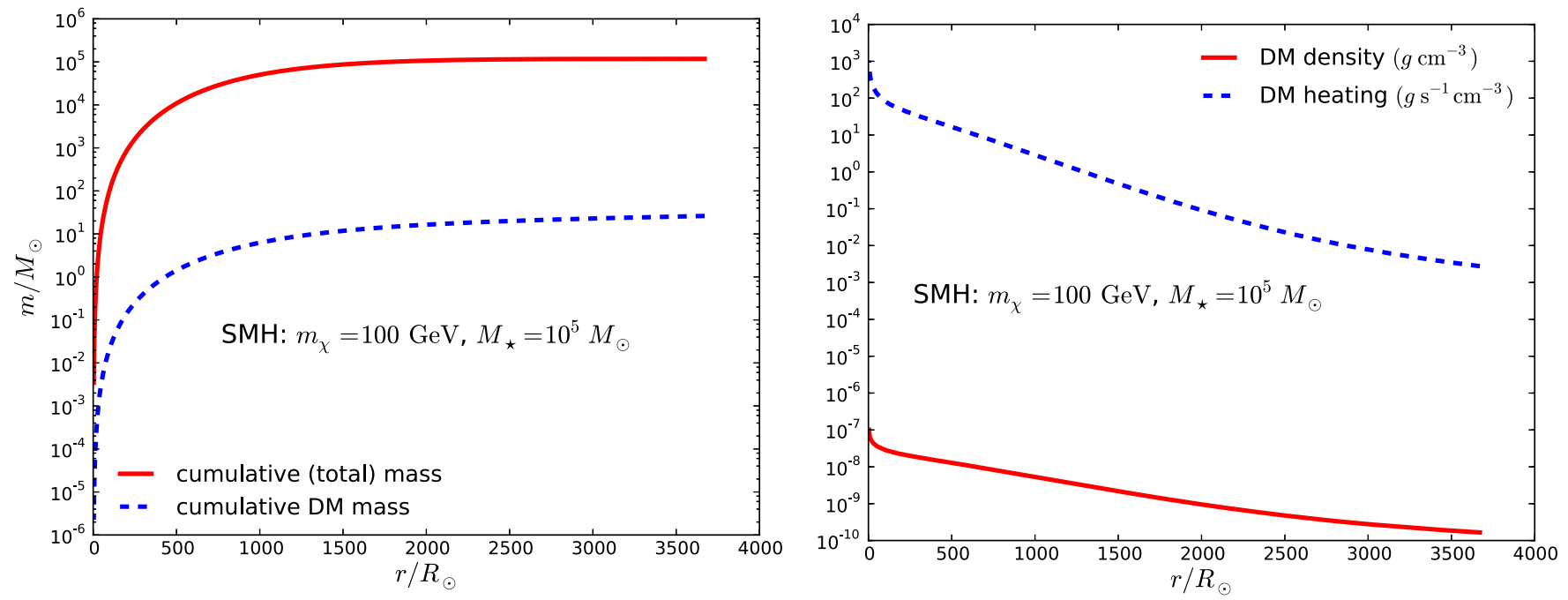

Figure 5. Cumulative mass profiles (left-hand plot) and DM heating (right-hand plot) for a DS of $10^{5} M_{\odot}$, residing in SMH and $m_{\chi}=100 \mathrm{GeV}$.

In Figure 3, we plot the luminosity at a given radius $L(r)$ and fraction of convective luminosity $L_{c}(r)$, as a function of stellar radius, for DSs with $10^{5} M_{\odot} . L_{c}(r)$ is the luminosity at a given radius within the star contributed by convection, i.e.,

$$
L_{c}(r)=L(r)-L_{\mathrm{rad}}(r),
$$

where $L_{\mathrm{rad}}(r)$ reflects the part due to diffusive radiation transfer. We can see that the fraction due to $L_{c}(r)$ is important in the centers and at the very edge of the stars, while $L_{\text {rad }}$ dominates throughout most of the interior. Thus, supermassive DSs are mostly dominated by the radiative transfer of energy, regardless of WIMP mass or accretion rate (see also Section 4). The importance of $L_{\text {rad }}$ increases with DS mass.

We also look at the distribution and amount of DM within DSs. To this end, we compile plots of the gas (i.e., baryonic) and DM density profiles for our fiducial $10^{5} M_{\odot}$ DS, for different halo environments and WIMP masses in Figure 4. The mass density in DM is roughly three orders of magnitude below the one for the baryonic mass density, showing how subdominant $\mathrm{DM}$ is compared to baryonic matter. The shape of both density profiles as well as their absolute magnitude agrees excellently with the results in Figure 3, case 1, of Spolyar et al. (2009), for DSs solely powered by DM heating (i.e., no fusion included), as is the case for our models. The shape of our density profiles is independent of the other parameters (halo and WIMP mass), while the densities are higher for the low-accretion environment $(\mathrm{SMH})$ at fixed WIMP mass, or for higher WIMP mass at fixed halo environment (in agreement with the plots of the central density in Figures 2, 8, and 9). As an illustrative corollary to these results, we show the cumulative mass profiles for SMH and $m_{\chi}=100 \mathrm{GeV}$ in the left-hand plot of Figure 5. We can see that, for a DS with $10^{5} M_{\odot}$, the mass in DM only amounts to roughly $20 M_{\odot}$, or $0.02 \%$ of the total stellar mass. The righthand plot of this same figure, on the other hand, shows how the amount of DM heating, as defined in Equation (2), follows the DM density profile within the DS.

\section{COMPARISON TO POLYTROPIC MODELS}

In this section, we present a detailed comparison of our results obtained using MESA with the polytropic models of Freese et al. (2010). We first point out that polytropes do indeed provide rather good approximations to true stellar models. However, there are some important differences to previous results that may impact observability.

First, we examine the case of SMH as defined in Equation (5). A comparison of the main stellar characteristics summarized in Table 1 with those in Table 1 of Freese et al. (2010) shows that, in the mass range of $10^{4}-10^{5} M_{\odot}$, our DSs are hotter by a factor of 1.41-1.51 than those in Freese et al. (2010), smaller in radius by a factor of $0.64-0.69$, denser by a factor of 3.00-3.41, and more luminous by a factor of 1.87-1.96. Thus, the overall colors of our DSs are not very different from the previous models, while our luminosities and central densities are significantly higher.

Next we examine the case of LMH as defined in Equation (6). DSs in LMHs are able to accrete more baryons and DM, and hence can grow more massive than in SMHs. A comparison of the main stellar characteristics of our Table 2 with Table 3 from Freese et al. (2010) reveals that the change in those parameters is consistent with the previous results for SMH: in the mass range of $10^{5}-10^{6} M_{\odot}$, our DSs are hotter by a factor of $1.45-1.67$ than those in Freese et al. (2010), smaller in radius by a factor of $0.60-0.62$, denser by a factor of 3.46-4.85, and more luminous by a factor of 1.98-2.19.

A closer examination of the stellar structure reveals further interesting comparison between the results of MESA and those assuming polytropes of Freese et al. (2010). First, we focus on the pressure distribution within the star. Assuming a polytropic law, $P / P_{c}=\left(\rho / \rho_{c}\right)^{1+1 / n_{\text {eff }}}$ with the central pressure $P_{c}$ and central density $\rho_{c}$, we solve for $n_{\text {eff }}$,

$$
n_{\mathrm{eff}}=\left[\frac{\log \left(P / P_{c}\right)}{\log \left(\rho / \rho_{c}\right)}-1\right]^{-1} .
$$

In the case of exact polytropic models, $n_{\text {eff }}$ is simply the usual polytropic index, ranging between $n=0, \ldots, 5$. Radiative stars are well approximated by $(n=3)$-polytropes, and DSs more massive than a few hundred solar masses have been found to follow ( $n=3$ )-polytropes to a good extent for much of their stellar interior (see Spolyar et al. 2009; Freese et al. 2010). It is thus instructive to plot relationship (11) for our models, i.e., for $P(r), P_{c}, \rho(r), \rho_{c}$, as calculated by MESA. This way, we can see where departures from a polytropic law are most pronounced, and which parts of the interior of a given supermassive DS are well approximated by $(n=3)$-polytropes. For $m_{\chi}=100 \mathrm{GeV}$, 

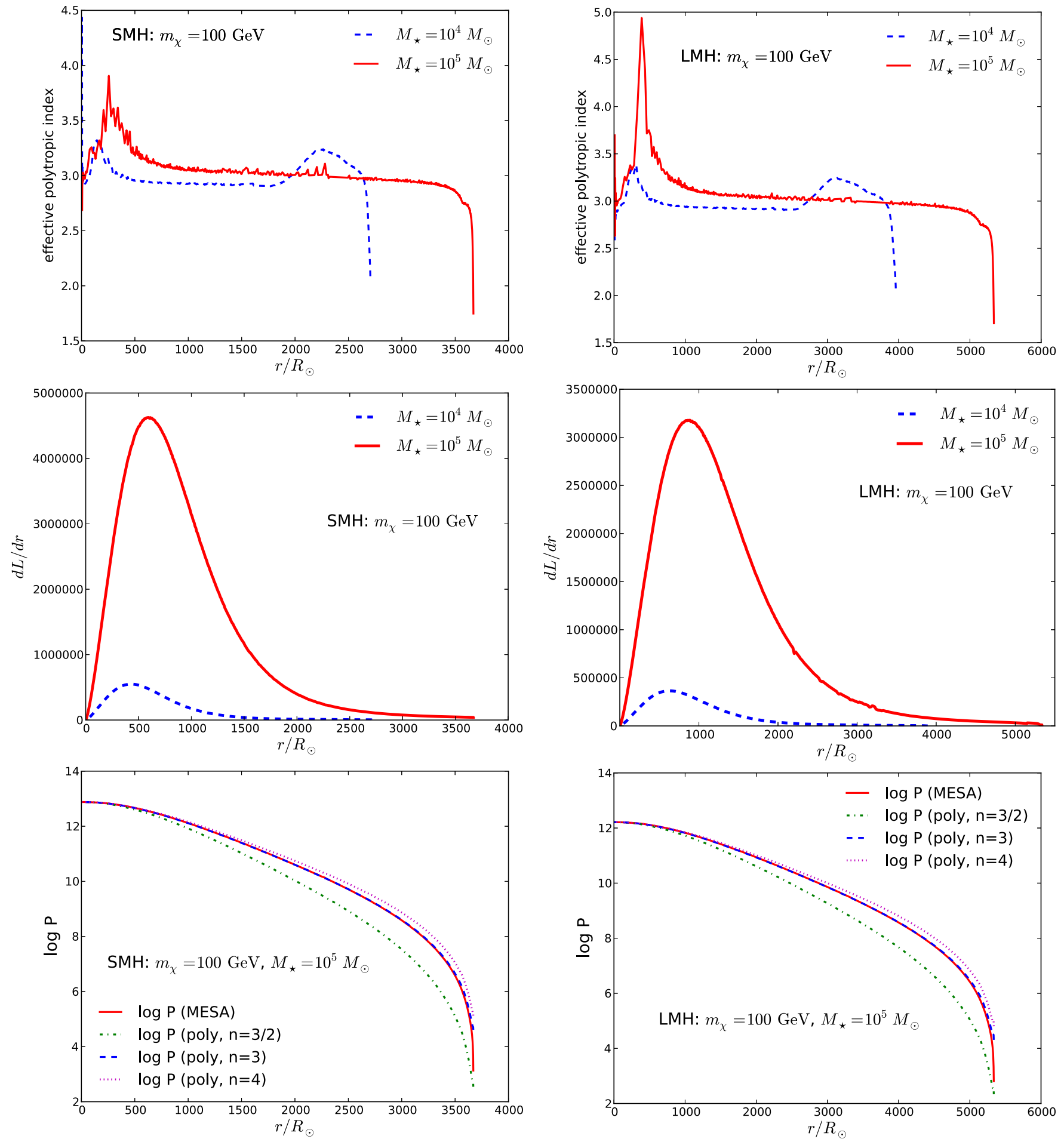

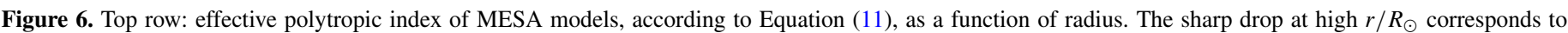

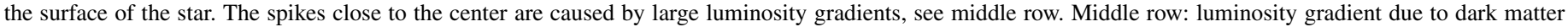

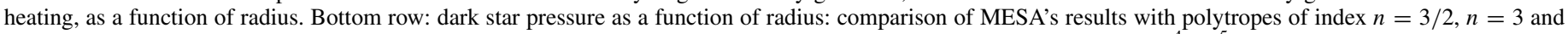

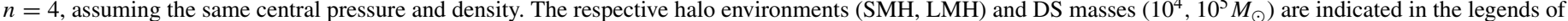

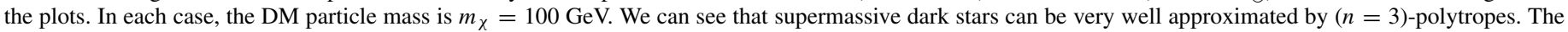
same plots for different WIMP masses can be found in Appendix A, Figures 10 and 11.

those results can be found in the top row of Figure 6. We can see that for supermassive DSs the effective polytropic index is, indeed, remarkably close to $n=3$ for most of the stellar interior, in accordance with Freese et al. (2010). Again, the "bump" close to the surface for the $10^{4} M_{\odot}$ model is caused by the high superadiabaticity gradients, which develop at masses above $100 M_{\odot}$. Without using MLT++, this bump would have been more pronounced (see also Section 3). The impact of superadiabaticity decreases again for higher stellar masses, as is evident in the curve for the $10^{5} M_{\odot}$ model. In summary, in the mass range $100-1000 M_{\odot}$, very inefficient convection with large superadiabatic gradients develop in the envelopes of these models. While the effective polytropic index of low-mass DSs with about $10-20 M_{\odot}$ is close to $n_{\text {eff }}=3 / 2$, this value 

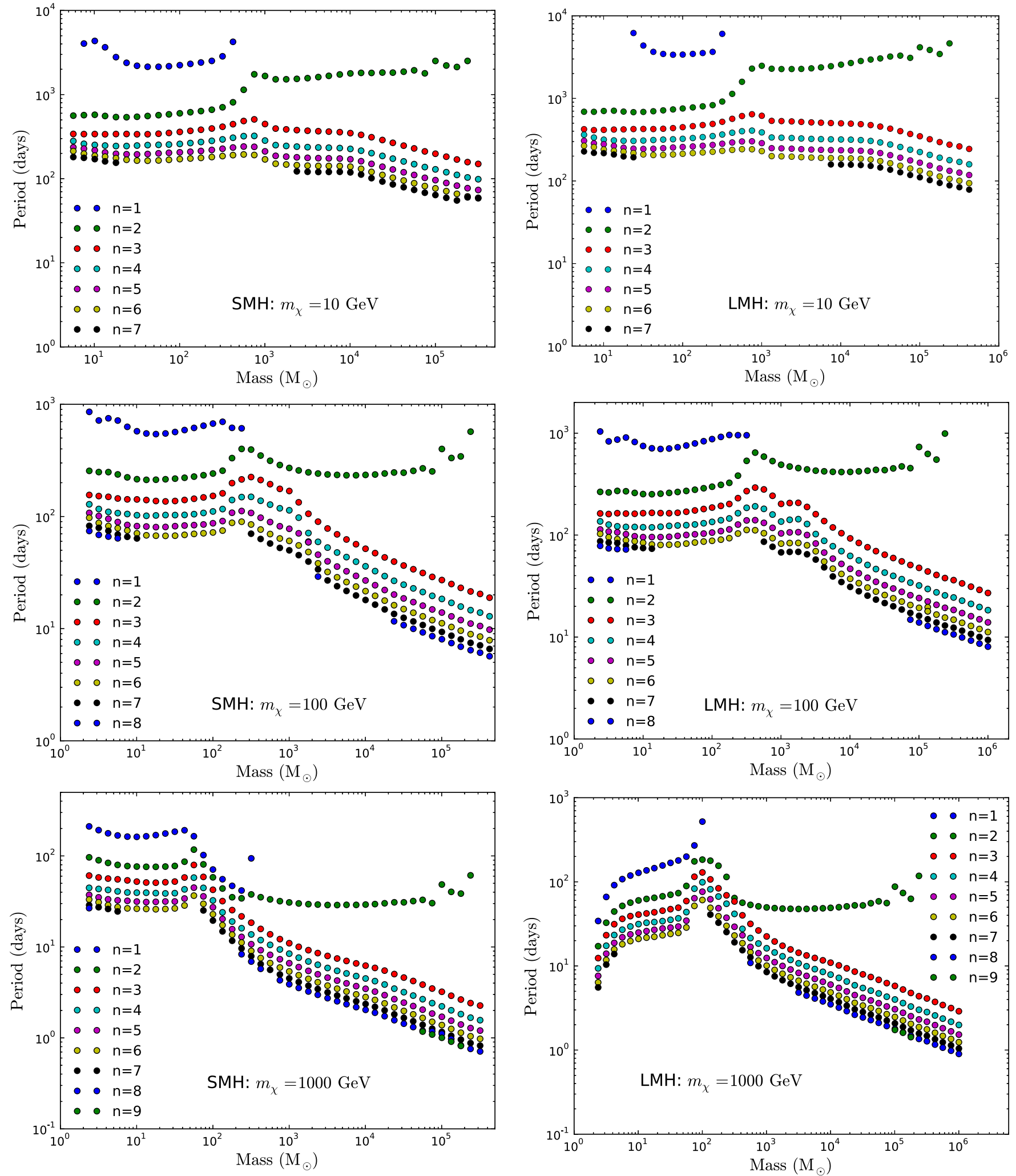

Figure 7. Radial, adiabatic pulsation periods as a function of DS mass for models with $m_{\chi}=10,100,1000 \mathrm{GeV}$ (top, middle, bottom row). Halo environments are indicated in the legends of the plots. The curves are for different overtone number, from $n=1$ (upper-most curve; the fundamental radial oscillation) to the highest overtone number in the respective plots (lower-most curve; the dots for $n=9$ lie close to the ones for $n=8$ in the bottom panel). The sharp feature at $\sim 100 M_{\odot}$ is a signature of the onset of superadiabaticity in the envelope, as discussed in the text.

steadily increases to above $n_{\text {eff }}=2$ for more than $100 M_{\odot}$. The numerical signature of our MLT++ prescription limiting the superadiabaticity happens when $L_{c} \sim L_{\mathrm{rad}}$, while $n_{\text {eff }}$ continues to approach the value of 3 for increasing DS mass. Then, $L_{\mathrm{rad}}$ becomes increasingly important. The corresponding proximity to $(n=3)$-polytropes is further illustrated in the bottom row of Figure 6, where we compare the radial run of the DS total pressure of our MESA results with polytropes of indices 
Table 3

Radial $(l=0)$ Pulsation Periods with Overtone Number $n$, for Dark Stars of Different Mass with $m_{\chi}=100 \mathrm{GeV}$ and $\dot{M}=10^{-3} M_{\odot} \mathrm{yr}^{-1}(\mathrm{SMH})$

\begin{tabular}{|c|c|c|c|}
\hline \multicolumn{4}{|c|}{$M_{\star}=10^{4} M_{\odot}: T_{\text {eff }}=15228.50 \mathrm{~K}, L_{\star}=3.423 \times 10^{8} L_{\odot}, R_{\star}=2660.48 R_{\odot}$} \\
\hline$n$ & $\begin{array}{c}f \\
(\mu \mathrm{Hz})\end{array}$ & $\begin{array}{l}\text { Periods } \\
\text { (days) }\end{array}$ & \\
\hline 2 & 0.04932 & 234.65 & \\
\hline 3 & 0.22455 & 51.54 & \\
\hline 4 & 0.32198 & 35.94 & \\
\hline 5 & 0.43076 & 26.87 & \\
\hline 6 & 0.53603 & 21.59 & \\
\hline 7 & 0.64288 & 18.00 & \\
\hline \multicolumn{4}{|c|}{$M_{\star}=10^{5} M_{\odot}: T_{\text {eff }}=24463.62 \mathrm{~K}, L_{\star}=4.150 \times 10^{9} L_{\odot}, R_{\star}=3589.72 R_{\odot}$} \\
\hline$n$ & $f(\mu \mathrm{Hz})$ & Periods (days) & Observer's Frame (days) \\
\hline 2 & 0.02896 & 399.69 & 6323.10 \\
\hline 3 & 0.42679 & 27.12 & 429.04 \\
\hline 4 & 0.62926 & 18.39 & 290.93 \\
\hline 5 & 0.83194 & 13.91 & 220.06 \\
\hline 6 & 1.03558 & 11.18 & 176.87 \\
\hline 7 & 1.23825 & 9.35 & 147.92 \\
\hline 8 & 1.43942 & 8.04 & 127.19 \\
\hline
\end{tabular}

Note. Periods are in the rest frame of the respective DS, unless otherwise indicated.

$n=3 / 2, n=3$, and $n=4$. Except for close to the surface, the pressure of our MESA models lies basically on top of the $(n=3)$-polytrope, showing again that supermassive DSs can be very well approximated by $(n=3)$-polytropes. We note that also in Freese et al. (2010), an interpolation between $n=1.5$ and $n=3$ was necessary for DSs beyond a few $100 M_{\odot}$. It is remarkable that we find the same behavior using MESA, given its capability to draw on elaborate equation-of-state tables.

Focusing again on the top row of Figure 6, we can see that the curve for the $10^{5} M_{\odot}$ model has a "spike" near the center. This deviation from a clean polytropic model is caused by the substantial release of heat due to the DM annihilation. In the middle row of Figure 6, we plot the differential change in luminosity, as a function of radius, i.e.,

$$
\frac{d L}{d r}=4 \pi r^{2} f_{Q} \hat{Q}_{\mathrm{DM}}
$$

(see Equation (3)). One can see that the "spike" in the effective polytropic index in the top panel coincides with the location of the maximal change in luminosity in the middle panel of the figure. The absolute values of $d L / d r$ increase with increasing WIMP mass, since DSs are smaller and hence denser for larger WIMP mass (see also Figures 1 and 4). The higher DM densities boost $d L / d r$, in turn.

In order to illustrate the robustness of the above results, we also show in Appendix A the corresponding plots for the cases of $m_{\chi}=10 \mathrm{GeV}$ and $m_{\chi}=1000 \mathrm{GeV}$ in Figures 10 and 11, respectively.

\section{ADIABATIC PULSATION PERIODS}

An interesting question of DS astrophysics is the possibility of pulsations. If DSs were found to pulsate, this could represent yet another observational distinction to other objects at high redshifts, such as the first galaxies and quasars. In this section, we only examine the representative range of pulsation frequencies of our DS models; we do not consider the more detailed question of the driving and damping of these pulsation modes.
Table 4

Radial $(l=0)$ Pulsation Periods with Overtone Number $n$, for Dark Stars of Different Mass with $m_{\chi}=100 \mathrm{GeV}$ and $\dot{M}=10^{-1} M_{\odot} \mathrm{yr}^{-1}(\mathrm{LMH})$

\begin{tabular}{|c|c|c|c|}
\hline \multicolumn{4}{|c|}{$M_{\star}=10^{4} M_{\odot}: T_{\text {eff }}=12538.20 \mathrm{~K}, L_{\star}=3.408 \times 10^{8} L_{\odot}, R_{\star}=3916.37 R_{\odot}$} \\
\hline$n$ & $f(\mu \mathrm{Hz})$ & Periods (days) & \\
\hline 2 & 0.02776 & 416.88 & \\
\hline 3 & 0.12461 & 92.88 & \\
\hline 4 & 0.18514 & 62.51 & \\
\hline 5 & 0.24784 & 46.70 & \\
\hline 6 & 0.31034 & 37.29 & \\
\hline 7 & 0.37280 & 31.05 & \\
\hline \multicolumn{4}{|c|}{$M_{\star}=10^{5} M_{\odot}: T_{\mathrm{eff}}=20311.98 \mathrm{~K}, L_{\star}=4.169 \times 10^{9} L_{\odot}, R_{\star}=5218.99 R_{\odot}$} \\
\hline$n$ & $f(\mu \mathrm{Hz})$ & Periods (days) & $\overline{\text { Observer's Frame (days) }}$ \\
\hline 2 & 0.01581 & 732.13 & 11677.47 \\
\hline 3 & 0.24257 & 47.71 & 760.97 \\
\hline 4 & 0.36104 & 32.06 & 511.36 \\
\hline 5 & 0.47971 & 24.13 & 384.87 \\
\hline 6 & 0.59866 & 19.33 & 308.31 \\
\hline 7 & 0.71624 & 16.16 & 257.75 \\
\hline 8 & 0.83278 & 13.90 & 221.70 \\
\hline \multicolumn{4}{|c|}{$M_{\star}=10^{6} M_{\odot}: T_{\text {eff }}=30976.24 \mathrm{~K}, L_{\star}=4.860 \times 10^{10} L_{\odot}, R_{\star}=7661.68 R_{\odot}$} \\
\hline$n$ & $f(\mu \mathrm{Hz})$ & Periods (days) & \\
\hline 3 & 0.42777 & 27.06 & \\
\hline 4 & 0.63034 & 18.36 & \\
\hline 5 & 0.83106 & 13.93 & \\
\hline 6 & 1.03377 & 11.20 & \\
\hline 7 & 1.23580 & 9.36 & \\
\hline 8 & 1.43755 & 8.05 & \\
\hline
\end{tabular}

Note. Periods are in the rest frame of the respective DS, unless otherwise indicated.

This allows us to treat the pulsations adiabatically, which greatly simplifies the calculation. The nonadiabatic calculation of the driving and damping of modes in these models will be presented in a forthcoming paper.

While supermassive DSs are mostly dominated by radiative transfer, as described in Section 3 and 4, we can see from Figure 3 that $L_{c}(r) / L(r) \gtrsim 0.1$, i.e., convection is not completely absent in supermassive DSs. Equivalently, looking at the square of the Brunt-Väisälä frequency defined as

$$
N^{2}=\frac{g}{r}\left[\frac{1}{\Gamma_{1}} \frac{d \ln P}{d \ln r}-\frac{d \ln \rho}{d \ln r}\right],
$$

with $\Gamma_{1} \equiv(d \ln P / d \ln \rho)_{\text {ad }}$, we find that it is negative throughout most of the DS interior, implying convective instability. We therefore do not expect to find gravity modes ("g-modes") in supermassive DSs.

On the other hand, acoustic modes, or p-modes, could be present in DSs. We calculated the adiabatic pulsation periods of radial modes (i.e., those for which $l=0$ ) with different overtone number $n$, where $n=1$ is the fundamental ("breathing") mode, and $n>1$ are higher overtone modes. ${ }^{8}$ Figure 7 shows the rest-frame pulsation periods for our DS models, covering the whole range of $\sim 10-10^{6} M_{\odot}$. We see that the $n=1$ modes disappear above DS masses of around $100 M_{\odot}$. We believe this

8 The pulsations were calculated using the MESA implementation of the ADIPLS code. We note that ADIPLS defines the order of the mode such that the lowest order radial oscillation has order $n=1$, contrary to the commonly used convention of assigning order 0 to the fundamental radial oscillation. For $p$-modes, $n>0$. 

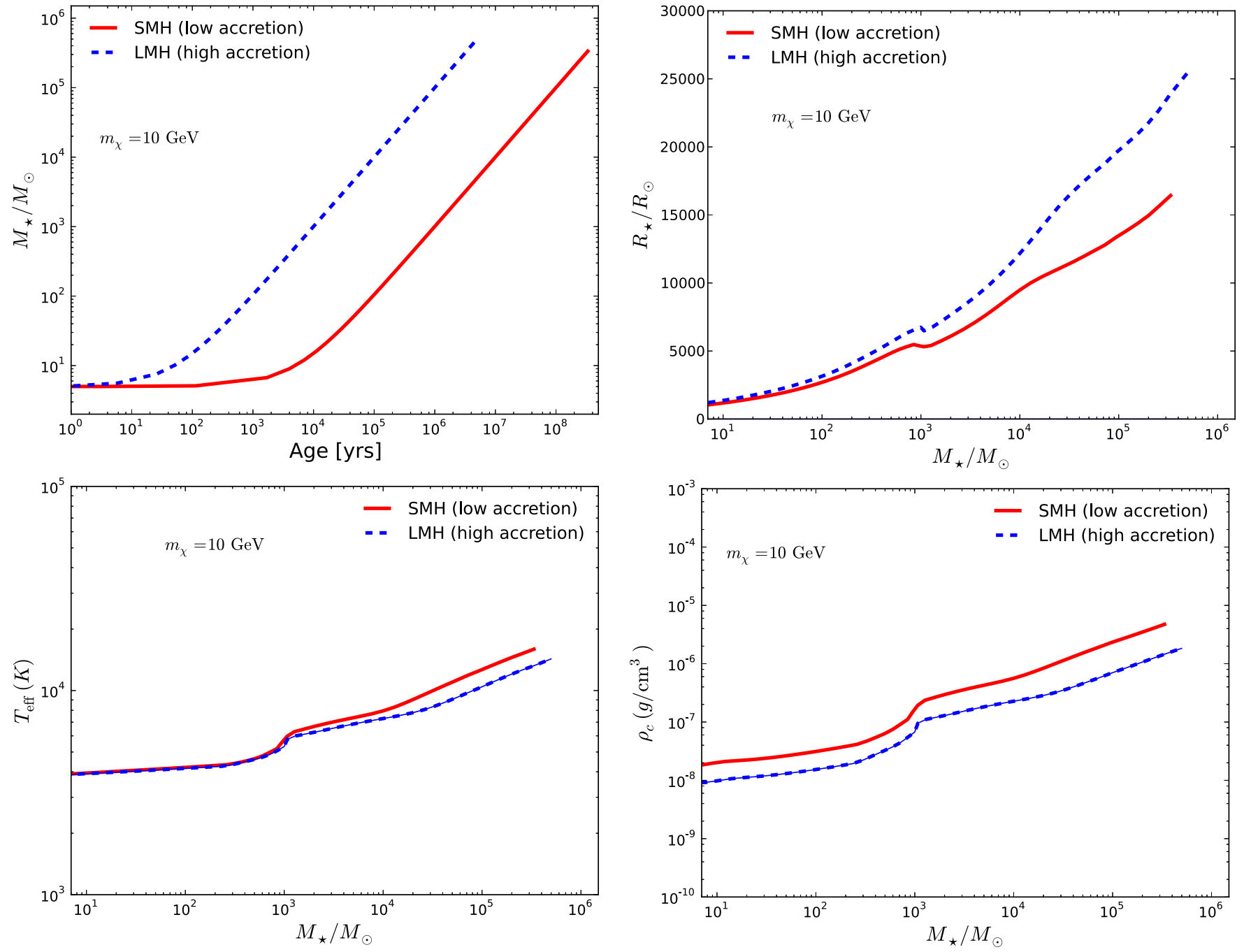

Figure 8. Same as Figure 2, but for $m_{\chi}=10 \mathrm{GeV}$.

is due to the change in energy transport from convection to radiation-domination above that mass range. Similarly, we note the change in slope in the plots for the periods around that same mass, again caused by the sharp transition to superadiabaticity and its suppression, which was also responsible for the "bump" in radius in Figure 9; as a reminder, this suppression is put in by hand to limit the growth of the superadiabatic gradients. As before, the transition is milder for small WIMP mass. As expected, the periods are much shorter for higher overtone number $n$. We also see that the periods are shorter for higher WIMP mass: while periods for high $n$ can span a range of 60-400 days for the $10 \mathrm{GeV}$ case, they are of order of days or less than a day for the $1000 \mathrm{GeV}$ case, in the rest frame. Also, more modes with high $n$ are excited for large WIMP mass.

In Tables 3 and 4, we give a more detailed list of the corresponding pulsation frequencies and periods for supermassive DSs in the range $10^{4}-10^{6} M_{\odot}$ for the $m_{\chi}=100 \mathrm{GeV}$ WIMP case. In this case, the pulsation periods lie in a range between 8 days and more than 2 years in the rest frame of the DSs. When converting to the observer's frame, one needs to multiply the periods by a factor of $\left(1+z_{\star}\right)$, where $z_{\star}$ denotes the redshift at which the DS under consideration has acquired its final mass. The time frame for this can vary tremendously, depending on the accretion rate (see left-hand upper plot in Figures 2, 8 and 9, re- spectively). This is independent of WIMP mass, however. A DS with $10^{5} M_{\odot}$ has an age of about $10^{8} \mathrm{yr}$ in a low accretion rate environment (SMH), in contrast to an age of about $10^{6} \mathrm{yr}$ in the high accretion rate environment $(\mathrm{LMH})$. For our adopted $\Lambda \mathrm{CDM}$ cosmology, this corresponds to redshifts of $z_{\star} \simeq 14.82(\mathrm{SMH})$ and $z_{\star} \simeq 14.95(\mathrm{LMH})$, respectively (compare these to the halo formation redshifts of $z=20(\mathrm{SMH})$ and $z=15(\mathrm{LMH})$ ). We include the converted periods for the case of a $10^{5} M_{\odot}$ DS in the tables, as well. The shortest periods to be expected in the observer's frame are given by the $1000 \mathrm{GeV}$ case, amounting to less than about 50 days, for modes with $n>6$.

Work is in progress to study the pulsations of these objects in more detail. In particular, we defer the nonadiabatic calculation of the possible driving and damping mechanisms for the pulsation modes to a future paper. Preliminary results suggest that the traditional $\kappa-\gamma$ mechanism could operate in these stars (see, e.g., Unno et al. 1989). A further source of driving could come from the DM itself. As the DS undergoes small perturbations, local changes in its baryonic density could lead to local changes in the DM density. This in turn would modulate the local DM heating rate. Depending on the size and relative phasing of these effects, this could be a source of driving for the pulsations. Of course, a much more quantitative approach is needed to assess the viability of this mechanism. We will study this and other possibilities in future work. 

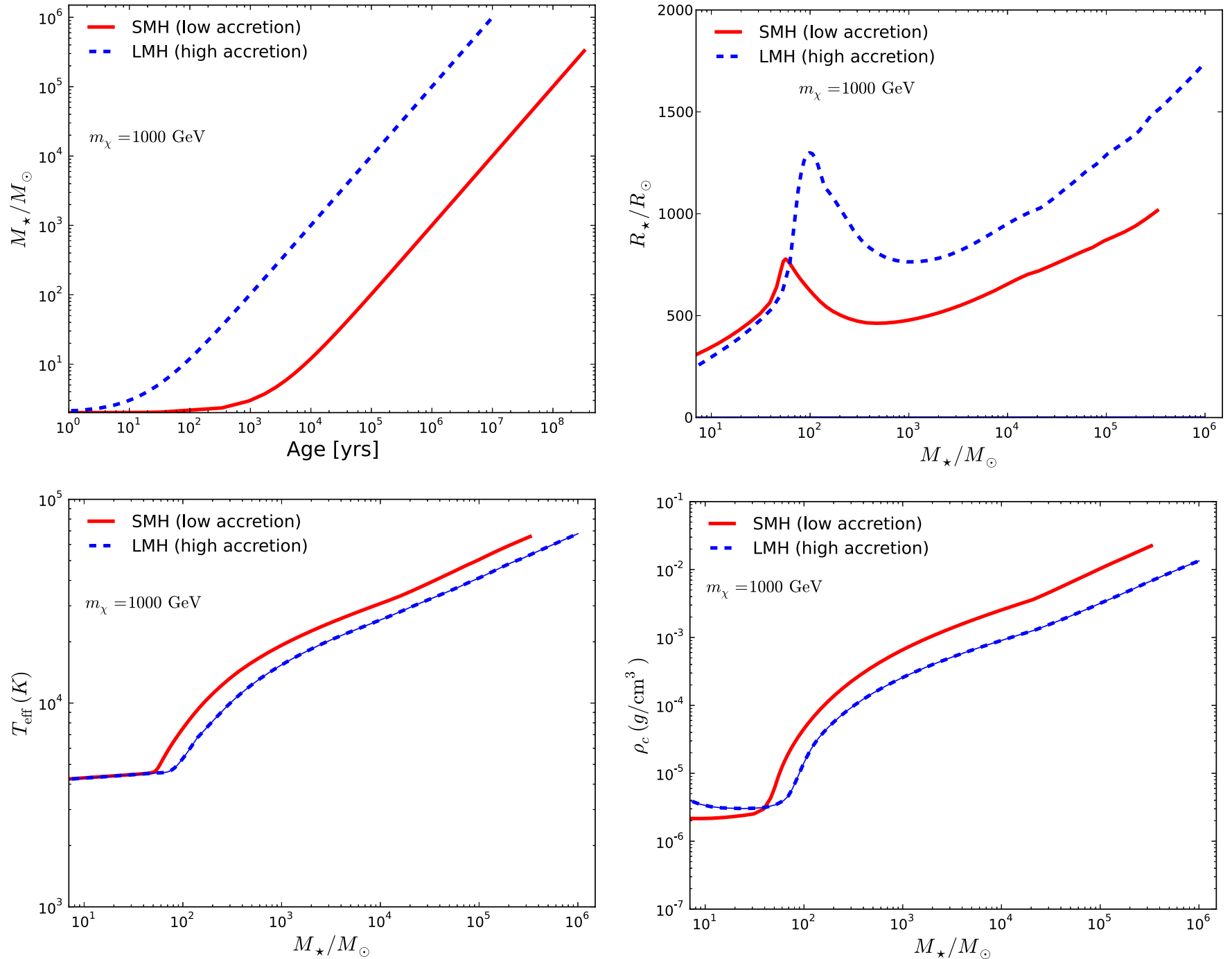

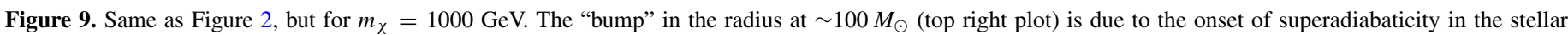
envelope, as explained in the text.

\section{CONCLUSIONS}

The bulk of this paper has been devoted to studying the properties of DSs using MESA, a fully fledged one-dimensional stellar evolution code that allows us to solve the stellar structure equations self-consistently, without any a priori assumptions on the equation of state, or other stellar characteristics. We were quite surprised how well the previous results using polytropes match the more accurate results using MESA. We have seen that supermassive DSs are extended, fluffy and cool objects, and in contrast to "normal" stars on the red giant branch, their lowdensity "envelopes" do not host an ultra-dense core. In fact, as we found, supermassive DSs can be very well approximated by $(n=3)$-polytropes, so their ratio of central to average density is not much different from a factor of about 54.

However, there are some differences between the results of MESA and previous polytropic models in the details, with positive implications for observability of DSs. We found that, in the mass range of $10^{4}-10^{5} M_{\odot}$, our DSs are hotter by a factor of 1.5 than those in Freese et al. (2010), are smaller in radius by a factor of 0.6 , denser by a factor of 3-4, and more luminous by a factor of 2. This increased luminosity should of course help in searches for DSs using the James Webb Space Telescope (see Zackrisson et al. 2010a, 2010b).
We also performed a first study of DS pulsations. While g-modes are excluded by the presence of convection in these models, radial and p-modes are permitted. We find that models of these stars pulsate on timescales that range from less than a day to more than two years in their rest frames, at a redshift of about 15 , depending on the DM particle mass and overtone number. The pulsation periods are significantly shorter for modes with high overtone number. In general, periods are also significantly shorter for higher WIMP mass: converting to the observer's frame, we find that the shortest periods are less than about 50 days, in the $1000 \mathrm{GeV}$ case for modes with $n>6$.

Work is in progress to study pulsations in more detail, including the novel idea of DM driven pulsations. As the DS undergoes small perturbations, changes in the local baryonic density could lead to changes in the local DM density, in turn modulating the local DM heating rate. Depending on the size and relative phasing of these effects, this could be a source of driving for the pulsations; a much more quantitative approach is needed to assess the viability of this mechanism. We will study this and other possibilities in future work. DS pulsations could someday be used to identify bright, cool objects uniquely as DSs. If the pulsations are detectable, then DSs could, in principle, also provide novel standard candles for cosmological studies. 

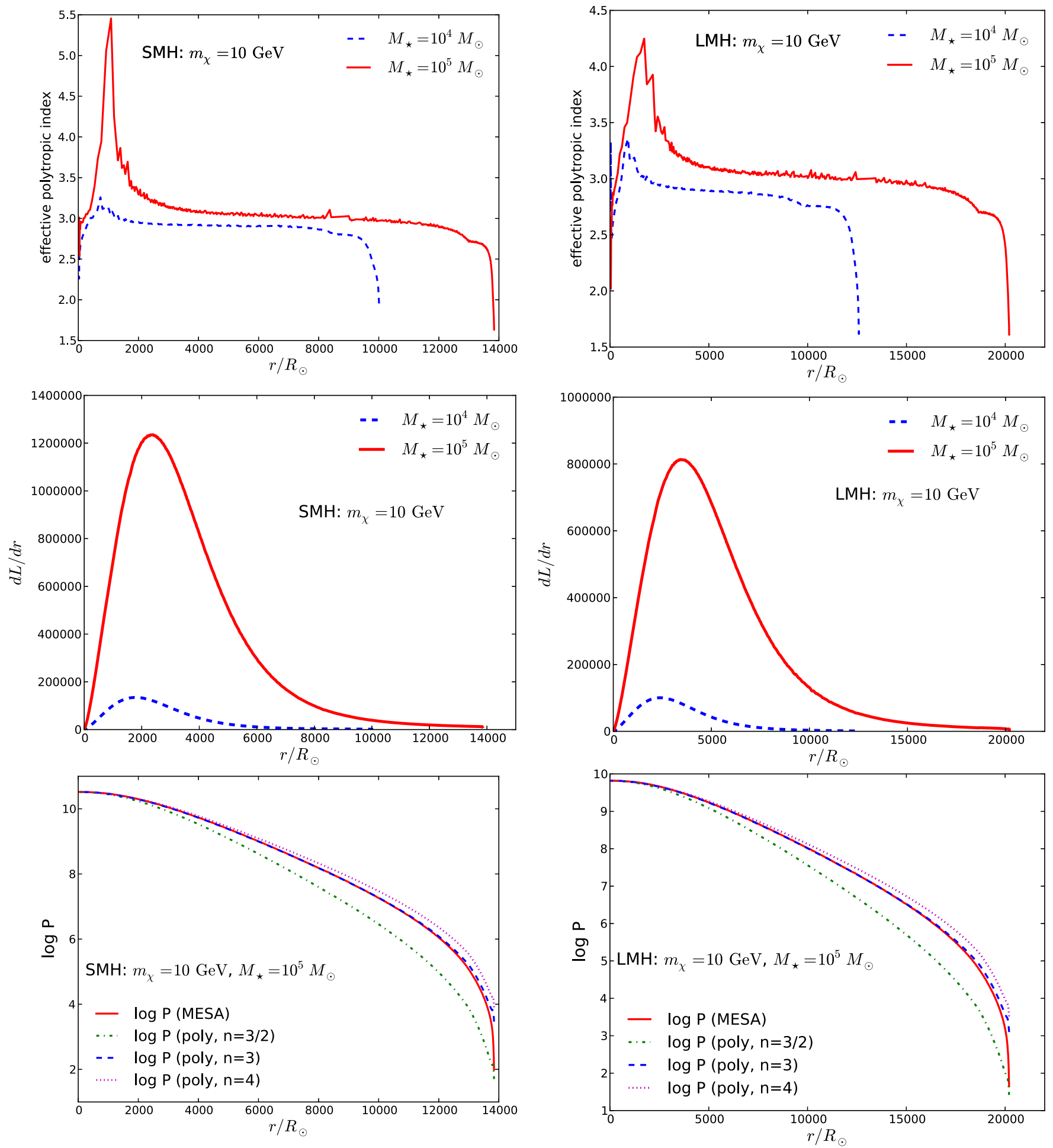

Figure 10. Same as Figure 6, but for $m_{\chi}=10 \mathrm{GeV}$.

We are grateful for helpful discussions with Douglas Spolyar, Peter Bodenheimer, Paul Shapiro, and Matthew Turk. T.R.D. thanks Josiah Schwab for valuable help with programming issues. T.R.D. and K.F. acknowledge the support by the Department of Energy under grant DOE-FG0295ER40899 and the Michigan Center for Theoretical Physics at the University of Michigan, Ann Arbor. M.H.M. and D.E.W. acknowledge the support of the National Science Foundation under grants AST-0909107 and AST-1312983, and M.H.M. acknowledges the support of NASA under grant NNX12AC96G. This work was supported in part by the National Science Foundation under grant PHYS-1066293 and the hospitality of the Aspen Center for Physics. The stellar evolution calculations presented in this paper were made using the MESA code (Paxton et al. 2011, 2013). The adiabatic pulsation calculations were made using the MESA implementation of the ADIPLS code (Christensen-Dalsgaard 2008). 

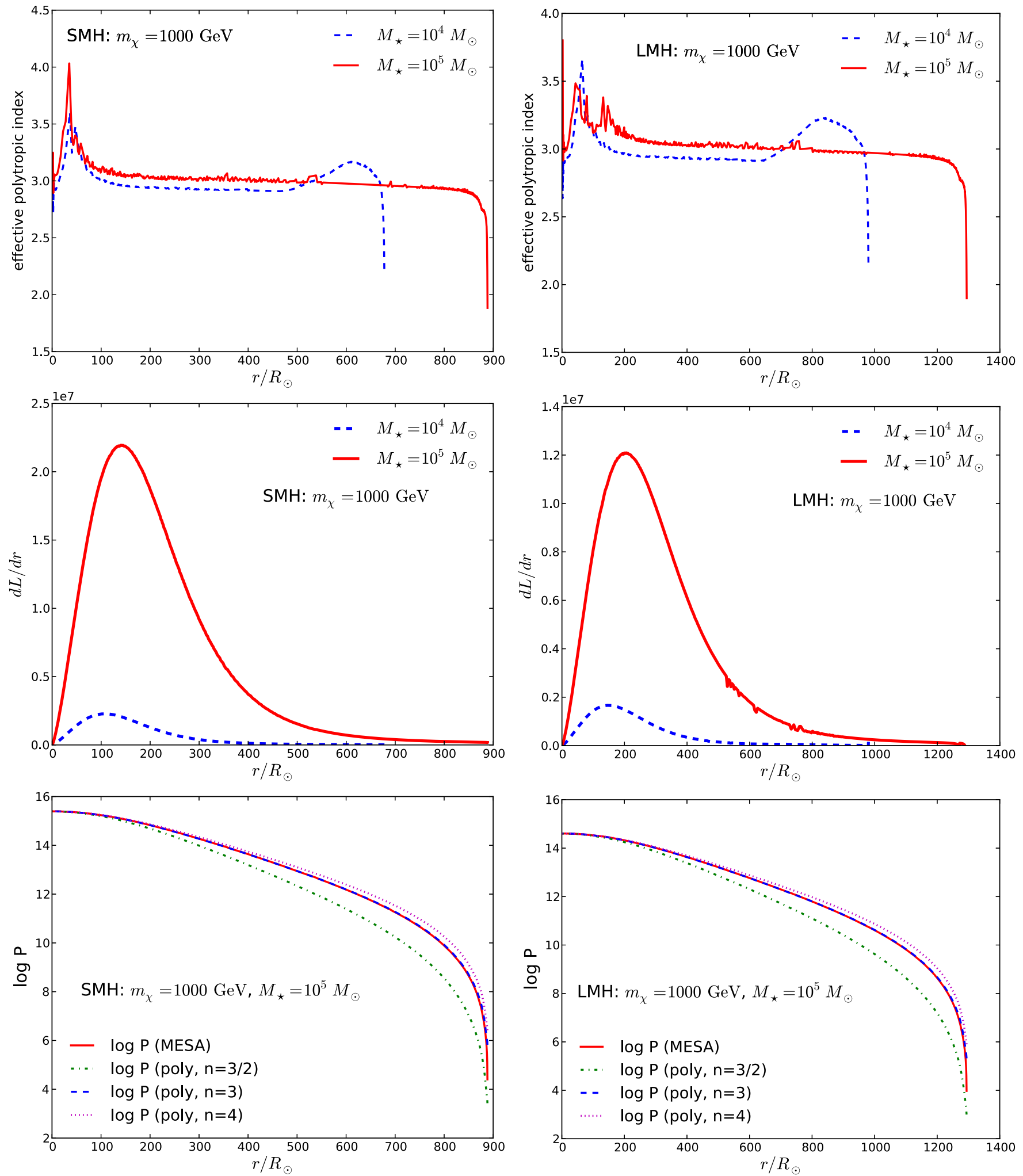

Figure 11. Same as Figure 6, but for $m_{\chi}=1000 \mathrm{GeV}$.

\section{APPENDIX A}

\section{DARK STAR PROPERTIES FOR DIFFERENT WIMP MASSES}

For better readability of the paper, we collect some of the plots of Section 3-4, for WIMP masses different from $100 \mathrm{GeV}$, in this section (Figures 8 and 9).

\section{APPENDIX B}

\section{DARK MATTER HEATING IN MESA}

The stellar evolution project MESA (Paxton et al. 2011, 2013) provides a number of tools that allow one to go beyond the standard picture of stellar evolution. For this study, we have used the other_energy_implicit module in MESA to include the 
energy deposited in the model due to DM annihilation. During a time step, this extra energy is added self-consistently to the model, in the same way that energy due to nuclear reactions would be. This code is included in a MESA run_star_extras file, which itself includes a Fortran program for calculating the DM heating rate, and the adiabatic contraction. The latter program has been used in Spolyar et al. (2009) and Freese et al. (2010), and has been provided to us from the authors for use in MESA. These files, along with the inlist file used for the stellar evolution calculations, can be found on http://mesastar.org/results.

We note that while the heating mechanism we investigate is due to the annihilation of DM particles, we do not assume that this leads to a depletion of DM. Rather, we assume that this DM is replenished due to a continuous infall of DM on centrophilic orbits within the minihalo. Thus, we adopt the same assumption as in Freese et al. (2010), the reference on which we have based our comparison between MESA's results and polytropes (see Section 4). In the code, we accomplish this by not removing (annihilated) DM from the reservoir.

Work is in progress to include the effects of DM capture via nuclei in the DS, as well as nuclear fusion. These mechanisms become important, once the DM "fuel" from adiabatic contraction and infall runs out. Then, the DS shrinks and stellar densities increase to high enough values for these mechanisms to set in, as shown in Spolyar et al. (2009). The results of this analysis will be presented in a future publication.

\section{REFERENCES}

Abel, T., Bryan, G. L., \& Norman, M. L. 2002, Sci, 295, 93

Ade, P. A. R., Aghanim, N., Armitage-Caplan, C., et al. (Planck Collaboration) 2014, A\&A, 571, A16

Ahn, K., \& Shapiro, P. R. 2007, MNRAS, 375, 881

Alvarez, M. A., Bromm, V., \& Shapiro, P. R. 2006, ApJ, 639, 621

Barkana, R., \& Loeb, A. 2001, PhR, 349, 125
Blumenthal, G. R., Faber, S. M., Flores, R., \& Primack, J. R. 1986, ApJ, 301,27

Bromm, V. 2013, RPPh, 76, 112901

Bromm, V., \& Larson, R. B. 2004, ARA\&A, 42, 79

Christensen-Dalsgaard, J. 2008, A\&SS, 316, 113

Clark, P. C., Glover, S. C.O., Klessen, R. S., \& Bromm, V. 2011, ApJ, 727, 110

Freese, K., Bodenheimer, P., Spolyar, D., \& Gondolo, P. 2008a, ApJL, 685, L101

Freese, K., Gondolo, P., Sellwood, J. A., \& Spolyar, D. 2009, ApJ, 693, 1563

Freese, K., Ilie, C., Spolyar, D., Valluri, M., \& Bodenheimer, P. 2010, ApJ, 716, 1397

Freese, K., Spolyar, D., \& Aguirre, A. 2008b, JCAP, 811, 014

Glover, S. 2013, in The First Galaxies, ed. T. Wiklind et al. (Astrophysics and Space Science Library, Vol. 396; Berlin: Springer), 103

Gondolo, P., Freese, K., Spolyar, D., \& Bodenheimer, P. 2013, arXiv:1304.7415

Greif, T. H., Bromm, V., Clark, P. C., et al. 2012, MNRAS, 424, 399

Hosokawa, T., Omukai, K., \& Yorke, H. W. 2011, ApJ, 756, 93

Hosokawa, T., Yorke, H. W., \& Omukai, K. 2010, ApJ, 721, 478

Ilie, C., Freese, K., \& Spolyar, D. 2011, NJPh, 13, 053050

Ilie, C., Freese, K., Valluri, M., Iliev, I. T., \& Shapiro, P. R. 2012, MNRAS, 422,2164

Iocco, F. 2008, ApJL, 677, L1

Navarro, J. F., Frenk, C. S., \& White, S. D. M. 1996, ApJ, 462, 563

Paxton, B., Bildsten, L., Dotter, A., et al. 2011, ApJS, 192, 3

Paxton, B., Cantiello, M., Arras, P., et al. 2013, ApJS, 208, 4

Ripamonti, E., Iocco, F., Ferrara, A., et al. 2010, MNRAS, 406, 2605

Shapiro, P. R., Iliev, I. T., \& Raga, A. C. 2004, MNRAS, 348, 753

Smith, R. J., Hosokawa, T., Omukai, K., Glover, S. C.O., \& Klessen, R. S. 2012a, MNRAS, 424, 457

Smith, R. J., Iocco, F., Glover, S. C.O., et al. 2012b, ApJ, 761, 154

Spergel, D. N., Bean, R., Doré, O., et al. 2007, ApJS, 170, 377

Spolyar, D., Bodenheimer, P., Freese, K., \& Gondolo, P. 2009, ApJ, 705, 1031

Spolyar, D., Freese, K., \& Gondolo, P. 2008, PhRvL, 100, 051101

Stacy, A., Greif, T. H., \& Bromm, V. 2010, MNRAS, 403, 45

Stacy, A., Pawlik, A., Bromm, V., \& Loeb, A. 2012, MNRAS, 421, 894

Stacy, A., Pawlik, A., Bromm, V., \& Loeb, A. 2014, MNRAS, 441, 822

Tan, J. C., \& McKee, C. F. 2004, ApJ, 603, 383

Turk, M. J., Abel, T., \& O'Shea, B. 2009, Sci, 325, 601

Unno, W., Osaki, Y., Ando, H., Saio, H., \& Shibahashi, H. 1989, Nonradial Oscillations of Stars (2nd ed.; Tokyo: University of Tokyo Press)

Yoshida, N., Abel, T., Hernquist, L., \& Sugiyama, N. 2003, ApJ, 592, 645

Zackrisson, E., Scott, P., Rydberg, C. E., et al. 2010a, ApJ, 717, 257

Zackrisson, E., Scott, P., Rydberg, C. E., et al. 2010b, MNRAS, 407, L74, (erratum 410, L57 [2011]) 